\title{
Measurements of top quark production cross-section at ATLAS
}

\author{
Akanksha Vishwakarma ${ }^{a}$, \\ On behalf of ATLAS Collaboration
}

${ }^{a}$ DESY, Germany

\begin{abstract}
Measurements of the inclusive and differential cross-sections for top quark pair and single top production crosssections in proton-proton collisions with the ATLAS detector at the Large Hadron Collider (LHC) are presented at centre-of-mass energies of $8 \mathrm{TeV}$ and $13 \mathrm{TeV}$. These measurements reach high precision and are compared to the best available theoretical calculations. The differential measurements, including results using boosted topology, probe our understanding of top quark pair production in the $\mathrm{TeV}$ regime. Measurements of top-quark pair with additional jets enable the study of effect of gluon radiation, especially the measurement with additional heavy flavour jets provides test of multi-scale QCD calculation. The first evidence of single top quark production in the $s$-channel is quoted. Interference of top quark pair and single top productions is investigated, and the single top production cross-section in association with a $Z$ boson is measured. Measurements of the properties of the Wtb vertex in single top quark production allow to set limits on anomalous couplings.
\end{abstract}

Keywords: top quark, cross-section, $t \bar{t}$, single top, ATLAS experiment

\section{Introduction}

Even after more than two decades from its discovery, the unique properties of the top quark are still intriguing the researchers. With its mass close to the electroweak symmetry breaking scale, it is the heaviest of all known particles of the Standard Model (SM) $\left(m_{t}=173.3 \pm 0.7 \mathrm{GeV}\right)$ [1]. This implies large couplings with the Higgs boson $\left(y_{t} \sim 1\right)$ and also with new particles predicted in physics beyond the SM (BSM). It is also considered as main decay channel of possible new resonances, therefore it may provide hints to new physics processes. Due to the short life time of the top quark, it decays before hadronization giving an opportunity to study a bare quark. Furthermore, top quark

\footnotetext{
*Talk given at 21st International Conference in Quantum Chromodynamics (QCD18), 2 July - 6 July 2018, Montpellier - FR

${ }^{* *}$ Copyright 2018 CERN for the benefit of the ATLAS Collaboration. CC-BY-4.0 license.

Email address: akanksha.vishwakarma@desy.de (Akanksha Vishwakarma)
}

production it a large background to many important and rare processes, therefore it is crucial to understand and model this process with great precision.

This overview presents recent measurements of single top and $t \bar{t}$ production cross-section performed by the ATLAS collaboration [2] using the 2015-2016 data at $\sqrt{s}=13 \mathrm{TeV}$ and 2012 data at $8 \mathrm{TeV}$.

\section{2. $t \bar{t}$ production cross-section}

At LHC, the dominant process of top quark pair production is via gluon fusion which contributes to $~ 90 \%$ at $\sqrt{s}=13 \mathrm{TeV}$ and only $\sim 10 \%$ comes from quark annihilation. Top quarks decay almost exclusively into a $W$ boson and $b$ quark. Hence, the final states of top quark depend on decay modes of the $W$ boson, viz. all-hadronic channel (both $W$ decay to quark-antiquark pair), lepton+jets channel (one $W$ decays to leptonneutrino and other to quark-antiquark pair) and dilepton channel (both $W$ decay to lepton-neutrino pair).

The all-hadronic channel has the highest branching frac- 


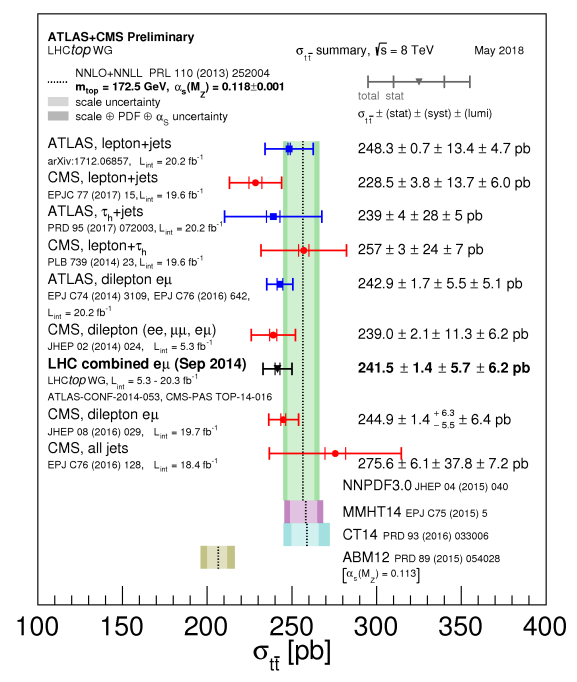

(a)

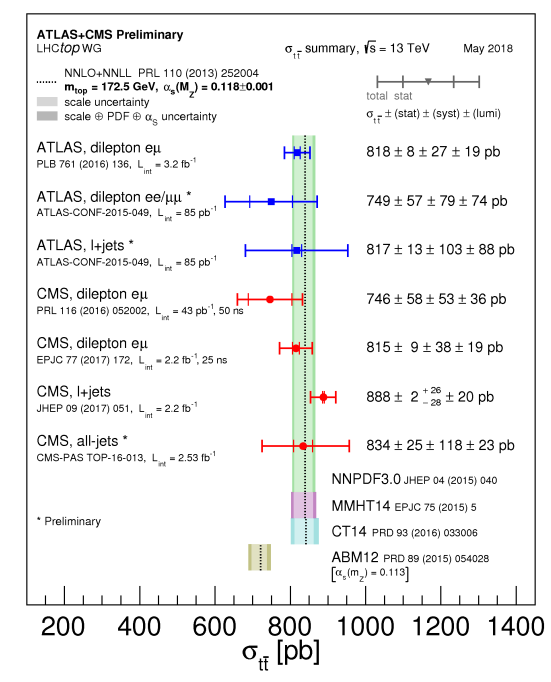

(b)

Figure 1: Summary of $t \bar{t}$ inclusive cross-section measured by the ATLAS (in blue) and CMS (in red) at (a) $8 \mathrm{TeV}$ and (b) $13 \mathrm{TeV}[3]$.

tion compared to the other decay channels but it suffers from large background. The dilepton channel features the lowest background contamination but also the smallest signal rate. The lepton+jets channel gives reasonably large samples and manageable backgrounds.

\subsection{Measurement of the inclusive cross-section}

The measurements of the $t \bar{t}$ inclusive cross-section $\left(\sigma_{t \bar{t}}\right)$ done by ATLAS and CMS are presented in the summary plot in figure 1 . The measurements are performed at different centre-of-mass energies, in different decay channels and are found to be in good agreement with the SM predictions within the uncertainties. Any deviation might suggest the manifestation of new physics.

The most precise measurement is performed in the $e \mu$ channel. It requires events with one electron and one muon of opposite charge. The numbers of events with exactly one or two $b$-tagged jets are counted and used to determine $\sigma_{t \bar{t}}$ as well as the efficiency to reconstruct and tag a $b$-jet from a top quark decay, hence reducing the systematic uncertainties on the signal yield significantly. The cross-section is measured in fiducial phase space, extrapolated to full phase space and compared with the theoretical predictions $\left(\sigma_{t \bar{t}}^{t h}\right)$ based on NNLO+NNLL QCD calculations. Table 1 quotes the results for data collected in 2012 with an integrated luminosity of $20.3 \mathrm{fb}^{-1}$ at $8 \mathrm{TeV}$ [4] and 2015 data with $3.2 \mathrm{fb}^{-1}$ at $13 \mathrm{TeV}$ [5]. The results obtained are consistent with the theoretical predictions.

\begin{tabular}{ccc}
$\sqrt{s}$ & $\sigma_{t \bar{t}}$ & $\sigma_{t \bar{t}}^{t h}$ \\
\hline $8 \mathrm{TeV}$ & $242.9 \pm 8.8 \mathrm{pb}$ & $252_{-13}^{+13} \mathrm{pb}$ \\
$13 \mathrm{TeV}$ & $818 \pm 36 \mathrm{pb}$ & $832_{-46}^{+40} \mathrm{pb}$
\end{tabular}

Table 1: Measured inclusive fiducial cross-section for the $t \bar{t}$ production in the $e \mu$ channel at 8 and $13 \mathrm{TeV}[4] 5]$. Results are compared with theoretical prediction.

The measurement is also performed in the lepton+jets channel at $8 \mathrm{TeV}$ using $20.3 \mathrm{fb}^{-1}$ data. The events are required to contain either one electron or one muon; the sample is then split into three signal regions with different jet multiplicities and number of $b$-tagged jets, in order to reduce the most relevant uncertainties coming from jet-energy scale and $b$-tagging efficiency. A neural network is used for better separation between signal and background. The results are obtained with a precision of 5.7\% [6] and are in agreement with the SM prediction as can be seen in equation 1 .

$$
\sigma_{t \bar{t}}=248.3 \pm 14.2 \mathrm{pb} \quad \sigma_{t \bar{t}}^{t h}=253_{-15}^{+13} \mathrm{pb}
$$

This analysis strategy improved the previous result which achieved a total uncertainty of $9.4 \%$ [7] using the same dataset.

\subsection{Measurement of the $t \bar{t}$ differential cross-section}

The differential cross-section measured as a function of different kinematic variables allows testing higher order perturbative QCD calculations. It is also used to validate state-of-the-art MC models and provide input for further developments. Distributions are measured at particle-level in the fiducial volume to facilitate the comparison with the theoretical models or extrapolated 


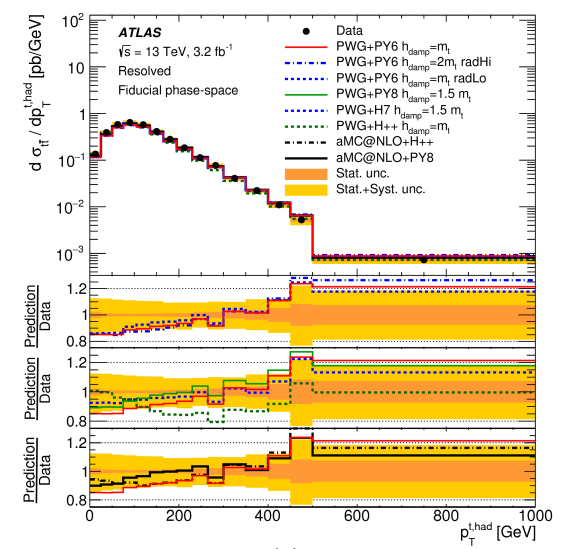

(a)

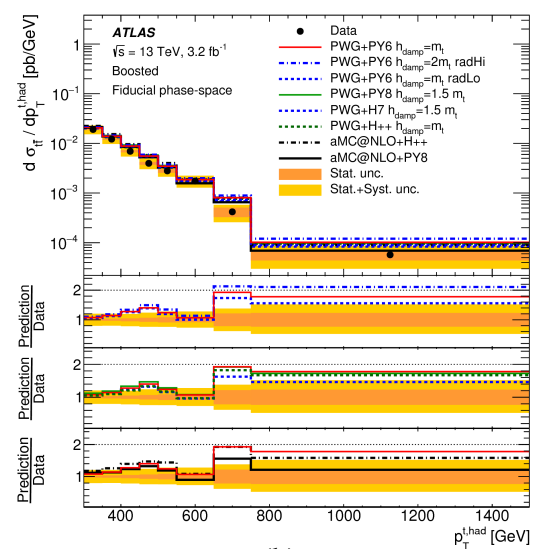

(b)

Figure 2: Relative differential cross-sections measured in a fiducial phase-space as a function of the transverse momentum of the hadronic top quark (a) in the resolved topology and (b) in the boosted topology, compared with the SM predictions obtained with different MC generators. The yellow bands indicate the total uncertainty of the data in each bin. The lower three panels show the ratio of these predictions to the data [8].

parton level. For this purpose the measured differential cross-sections obtained at reconstruction level are unfolded by correcting for the detector acceptance, efficiency and resolution effects.

The differential cross-sections are measured by the ATLAS collaboration as a function of the transverse momentum $\left(p_{\mathrm{T}, t}\right)$ and absolute rapidity $\left(\left|\mathrm{y}_{t}\right|\right)$ of the top quark, and of the transverse momentum $\left(p_{\mathrm{T}, t \bar{t}}\right)$, absolute rapidity $\left(\left|\mathrm{y}_{t \bar{t}}\right|\right)$ and invariant mass $\left(m_{t \bar{t}}\right)$ of the $t \bar{t}$ system.

In the lepton+jets channel two separate selections are applied to focus on different top quark momentum regions. In the resolved topology the decay products of the top quark are well separated and can be reconstructed individually. The events should contain at least one lepton and at least four jets, two of which must be $b$-tagged. The boosted topology probes the QCD in the $\mathrm{TeV}$ range by looking into high momentum top quarks. In this case the decay products tend to collimate and hence are reconstructed as single large radius jets. The events require at least one lepton, at least one small- $R$ jet and at least one large- $R$ jet. The analysis uses $3.2 \mathrm{fb}^{-1}$ of $13 \mathrm{TeV}$ data [8]. In general, the MC predictions agree with data in a wide kinematic region in both topologies. However, the shape of the particle level $\left(p_{\mathrm{T}, t}\right)$ distribution of hadronically decaying top quarks is poorly modelled by all NLO+PS predictions with larger disagreement at high $p_{\mathrm{T}}$, as can be seen in figure 2 .

A measurement is also done in the all-hadronic channel at particle level which is also extrapolated to parton level. It uses boosted topology and selects the

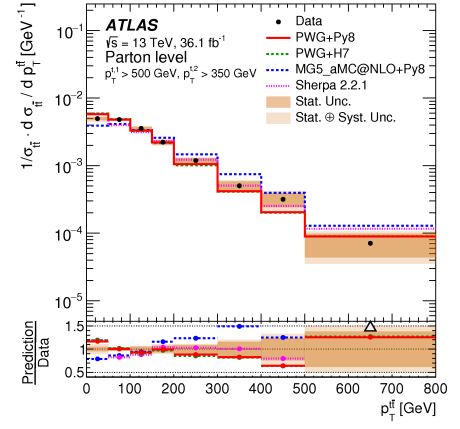

Figure 3: The normalized parton-level differential cross-sections as a function of transverse momentum of $t \bar{t}$ system. The orange bands indicate the total uncertainty in the data in each bin [9].

events having two high $p_{\mathrm{T}}$ large-radius jets in the final state. The data set corresponds to $36.1 \mathrm{fb}^{-1}$ [9]. Most predictions describe the data well with an exception of aMC@NLO + Pythia8, as shown in figure 3.

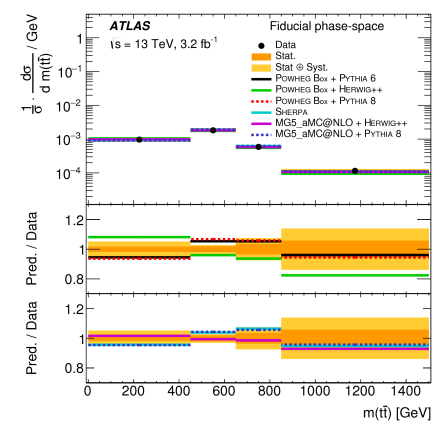

Figure 4: The measured normalised fiducial differential cross-sections as a function of invariant mass of $t \bar{t}$ system compared with the SM predictions obtained with different MC generators. The yellow bands indicate the total uncertainty of the data in each bin [10].

In the $e \mu$ channel, $t \bar{t}$ events are selected by requiring one opposite charge $e \mu$ pair, and at least two jets, one of which must be $b$-tagged. The measured differential 


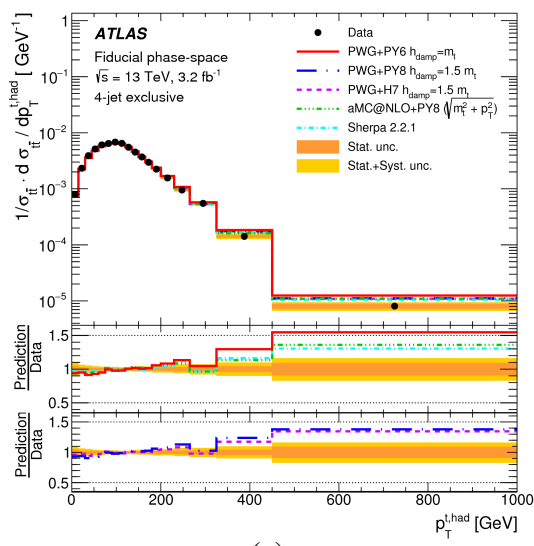

(a)

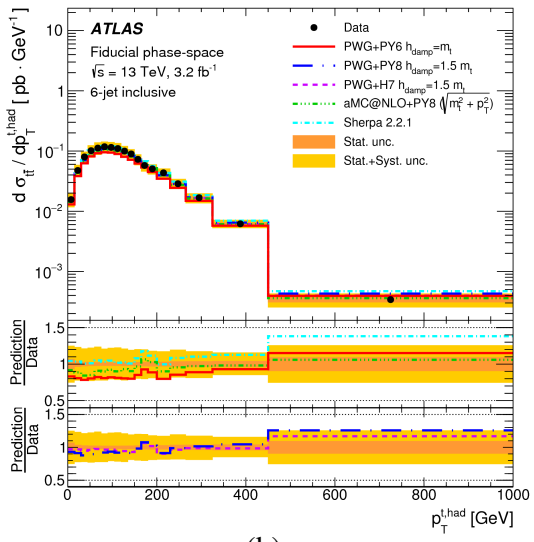

(b)

Figure 5: Normalised differential cross sections in the fiducial phase space as a function of $p_{\mathrm{T}}^{t \text {, had }}$ (a) in the 4-jet exclusive configuration (b) 6-jet inclusive configuration. The shaded area represents the total statistical and systematic uncertainties [11].

cross-sections as a function of above mentioned distributions are compared to predictions of NLO generators matched to parton showers and the measurements are found to be consistent with all models within the experimental uncertainties with the exception of the POWHEG+HERWIG++ predictions as demonstrated in figure 4. This measurement is performed using $3.2 \mathrm{fb}^{-1}$ of $13 \mathrm{TeV}$ data [10].

\subsection{Differential cross-section for $t \bar{t}+$ additional jets}

Top-quark pair production final states in $p p$ collisions often include additional jets not directly produced in the top quark decays. These arise mainly from hard gluon emissions and are described by QCD. These studies test the predictions of extra jet production and to provide data to adjust free parameters of the models to optimize their predictions.

\subsection{1. $t \bar{t}+n$-jets in $l+$ jets channel}

This measurement is performed using 2015 data $\left(3.2 \mathrm{fb}^{-1}\right)$ at $13 \mathrm{TeV}[11]$. The events are selected by a basic lepton+jets requirement and are categorized in three mutually exclusive samples depending on the number of additional jets. Study done in these fixed $\mathrm{n}$-jets configurations provides a better understanding of the effect of gluon radiation on $t \bar{t}$ kinematic variables than differential cross-sections inclusive in the number of jets. The measured ( $p_{\mathrm{T}}^{t \text {, had }}$ ) distribution in the 4-jet configuration is underestimated by the predictions at low values and overestimated at high values; this tendency of the predictions is reduced at higher jet multiplicity as described in figure 5 .

\subsection{2. $t \bar{t}+$ additional jets in e $\mu$ channel}

This analysis uses $3.2 \mathrm{fb}^{-1} 13 \mathrm{TeV}$ data. Events require opposite sign $e \mu$ pair with two $b$-tagged jets in the final state [12]. The normalised differential cross-

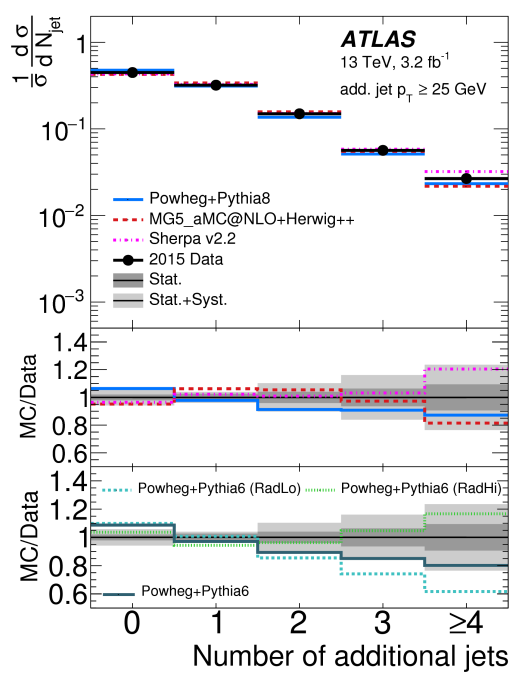

Figure 6: Unfolded jet multiplicity distribution of additional jets compared to different MC predictions. The middle and bottom panels show the ratios of different MC predictions of the normalised crosssection to the measurement and the ratios of Powheg+Pythia6 predictions with variation of the $\mathrm{QCD}$ radiation to the measurement, respectively. The shaded regions show the statistical uncertainty (dark grey) and total uncertainty (light grey) [12].

sections of $t \bar{t}$ production are presented as functions of the additional-jet multiplicity, as can be seen in figure 6 , and $p_{\mathrm{T}}$. While the kinematics of the jets from top quark decays are well described, the generators exhibit various levels of agreement with the measurements of observables that depend on the production of additional jets.

\subsection{3. $t \bar{t}+$ heavy-flavour jets in e $\mu$ and $l+$ jets channel}

Differential measurement of $t \bar{t}$ in association with heavy flavour jets is conducted using $36.1 \mathrm{fb}^{-1}$ of 


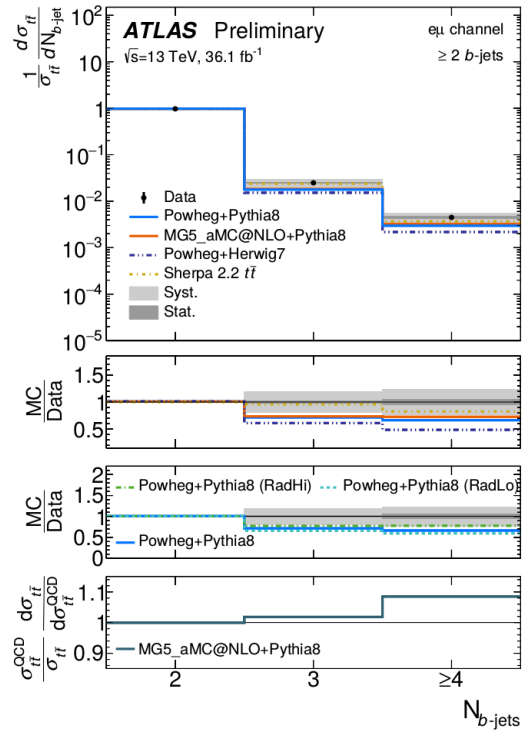

(a)

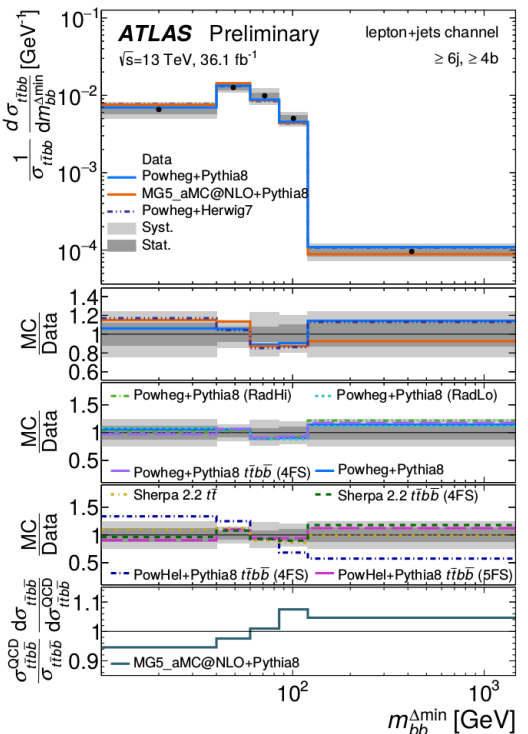

(b)

Figure 7: Relative differential cross-section measured in a fiducial phase-space as a function of (a) the $b$-jet multiplicity in $e \mu$ channel and (b) the invariant mass of the the two closest $b$-jets in $\Delta R$ in $l+$ jets channel and compared to various MC generators [13].

$13 \mathrm{TeV}$ data [13]. This measurement facilitates testing multi-scale calculations in QCD because of the nonnegligible mass of the $b$ quarks. In addition it constraints the main background to the interesting final state $t t H(b b)$. In general, the different observables are measured with a precision of $10 \%$ in most of the phase space, rising to $30 \%$ at the edge of the phase space for some of the observables. Figure 7 depicts the $b$-jet multiplicity in $e \mu$ channel and the invariant mass of the $b$-jet pair with minimum $\Delta R$.

\section{Single top cross-section}

\subsection{Inclusive cross-section}

The inclusive single top production cross-section is measured by ATLAS in all three production channels ( $t$-channel, $s$-channel and $t W$ production) at $\sqrt{s}=7,8,13 \mathrm{TeV}$ [14-18]. The results obtained are in good agreement with SM predictions. Figure 8 shows a summary of these measurements by ATLAS and CMS.

The evidence for single top quark production in the $s$-channel is presented using $20.3 \mathrm{fb}^{-1}$ of $8 \mathrm{TeV}$ data [16]. The analysis is performed on events containing one isolated electron or muon, large missing transverse momentum and exactly two $b$-tagged jets in the final state. The signal is extracted from the data utilizing a profile likelihood fit. A signal significance

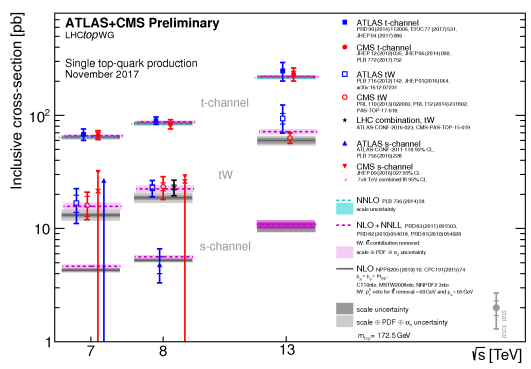

Figure 8: Summary of ATLAS and CMS measurements of the single top production cross-sections in various channels as a function of the centre of mass energy. The measurements are compared to theoretical calculations based on: NLO QCD, NLO QCD complemented with NNLL resummation and NNLO QCD (t-channel only) [3].

of 3.2 standard deviations is observed leading to a measured cross-section of $\sigma_{s}=4.8 \pm 0.8$ (stat. $)_{-1.3}^{+1.6}$ (syst.) $\mathrm{pb}$, which is consistent with the SM expectation. The expected significance for the analysis is 3.9 standard deviations.

\subsection{Differential cross-section}

Differential cross-sections are also measured in the $t$-channel at $8 \mathrm{TeV}\left(20.2 \mathrm{fb}^{-1}\right)$ [14] and $t W$ production at $13 \mathrm{TeV}\left(36.1 \mathrm{fb}^{-1}\right)$ [19]. The $t W$ production measurement is performed in the dilepton channel using a Boosted Decision Tree, in order to separate the signal from the $t \bar{t}$ background. The observables unfolded are the energy and mass of the lepton, $b$-jet or 


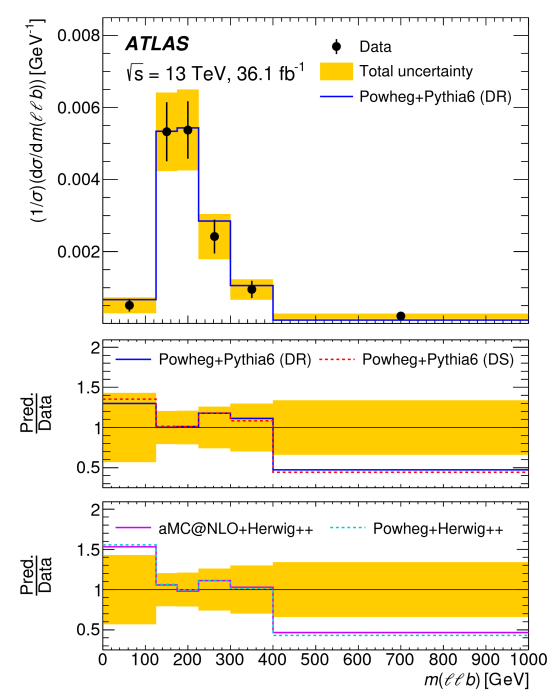

Figure 9: Normalised differential cross-sections unfolded from data, compared with selected MC models, with respect to $\mathrm{m}(l l b)$. Data points are placed at the horizontal centre of each bin [19].

their system. Most MC predictions are in agreement, but substantial negative slope is observed in some distributions, figure 9 for instance.

The $t$-channel has been measured in the $l+$ jets topology, making use of maximum likelihood fit of a multivariate Neural Network discriminant trained with sensitive variables. Differential cross-section as a function of the $p_{\mathrm{T}}$ and rapidity of the top quark at particle level and parton level are presented. The SM predictions provide good descriptions of the data.

\subsection{Interference between $t W b$ and $t \bar{t}$}

Normalized differential cross-section of the $t \bar{t}+t W b$ is measured in a fiducial phase- space region where interference effects between these topologies ( $t \bar{t}$ production and associated production of a single top quark with a $W$ boson and a $b$-quark) are significant [20]. Events with exactly two leptons and two $b$-tagged jets are selected from $36.1 \mathrm{fb}^{-1}$ of $13 \mathrm{TeV}$ data. The result as a function of invariant mass of a $b$-jet and a lepton is presented in figure 10 .

The standard prescriptions for interference modeling are significantly different from each other but are within $2 \sigma$ of the data. Both data removal (DR) and data subtraction (DS) schemes are compared. These results provide an important constraint on interference models and will guide future MC developments and tuning.

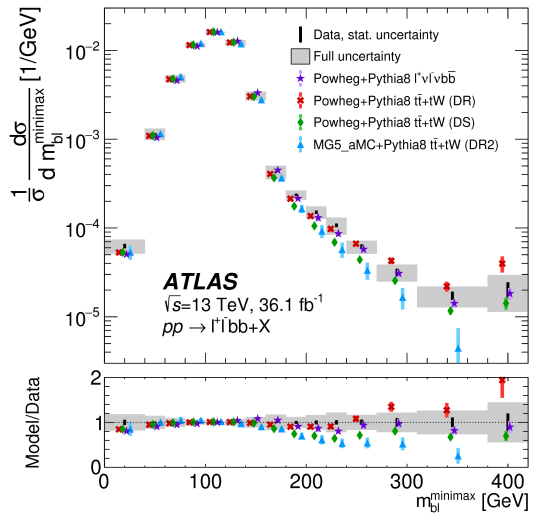

Figure 10: The unfolded normalized differential $m_{b l}^{\operatorname{minimax}}$ crosssection compared with theoretical models of the $t \bar{t}+t W b$ signal with various implementations of interference effects. The uncertainty of each data point includes all statistical and systematic sources, while uncertainties for each of the MC predictions correspond to variations of the PDF set and renormalization and factorization scales [20].

\subsection{Single top in association with $Z$ boson}

The production of a top quark in association with a $Z$ boson is investigated with $36.1 \mathrm{fb}^{-1}$ of $13 \mathrm{TeV}$ data [21]. The increased energy and bigger data collection enables to study this rare SM phenomena. Events containing three identified leptons and two jets, one of which is $b$-tagged are selected. A neural network is used to improve the background rejection and extract the signal. The resulting significance is $4.2 \sigma$ in the data and the expected significance is $5.4 \sigma$. The measured cross-section is $600 \pm 170$ (stat.) \pm 140 (syst.) fb. This result is in agreement with the predicted SM cross-section, calculated at NLO of $800_{-7.4 \%}^{+6.1 \%} \mathrm{fb}$.

\subsection{Wtb : probe for anomalous coupling}

The properties of the $W t b$ vertex determines the electroweak production and subsequent decay of single top quarks in the $t$-channel, which is described by the complex parameters of an effective Lagrangian as follows in equation 2

$$
\begin{aligned}
\mathcal{L}_{\text {eff }}= & -\frac{g}{\sqrt{2}} \bar{b} \gamma^{\mu}\left(V_{L} P_{L}+V_{R} P_{R}\right) t W_{\mu}^{-} \\
& -\frac{g}{\sqrt{2}} \bar{b} \frac{i \sigma^{\mu v} q_{v}}{m_{W}}\left(g_{L} P_{L}+g_{R} P_{R}\right) t W_{\mu}^{-} \\
& + \text {h.c. }
\end{aligned}
$$

In the SM at LO, all coupling constants vanish, except $V_{L}=V_{t b}$, which is a quark-mixing element in the CKM matrix. Deviations from these values would provide hints of BSM physics, and furthermore, complex values could imply $\mathrm{CP}$-violation. Analysis of a 
triple-differential decay rate in this channel can be used to determine the five generalised helicity fractions and phases, as well as the polarisation of the produced top quark and the complex parameters can then be constrained. This analysis [22] is based on $20.2 \mathrm{fb}^{-1}$ of $8 \mathrm{TeV}$ data, and the following limits at 95\% CL are derived on the coupling parameters as quoted in equation 3 .

$$
\begin{aligned}
& \operatorname{Re}\left[g_{R} / V_{L}\right] \in[0.12,0.17] \\
& \operatorname{Im}\left[g_{R} / V_{L}\right] \in[0.07,0.06]
\end{aligned}
$$

Also the polarisation $(P)$ of single top quarks in the $t$ channel production is constrained to be $P>0.72$.

\section{Summary}

The ATLAS collaboration performed several $t \bar{t}$ and single top cross-section measurements providing stringent tests of the SM. All the current results are posted at the top public result webpage [23]. The newest results, like the differential cross-section of $t \bar{t}$ with heavy flavour jets and the inclusive cross-section of single top in association with a $Z$ boson, are made possible with the increased integrated luminosity. All the measurements agree very well with the SM predictions within uncertainties.

However, in the case of $t \bar{t}$ differential cross-section, some of the models show discrepancies in some regions of phase space and further improvements are required. Most of these measurements are affected by systematics uncertainties like modeling uncertainty, $b$-tagging and jet energy scale uncertainties. For the single top measurements, the most important background comes from top quark pair production.

Further data collected in the upcoming years, with increased luminosity, are expected to improve the precision of statistically limited results. Time and new data will also allow for an improved understanding of systematic effects.

\section{References}

[1] The ATLAS, CDF, CMS, D0 Collaborations, arxiv:1403.4427 hep-ex]

[2] ATLAS Collaboration, JINST, 3 (2008) S08003

[3] ATLAS Collaboration, Summary plots from the ATLAS Top physics group https://atlas.web.cern.ch/Atlas/GROUPS/PHYSICS/CombinedSummaryPlots/TOP/

[4] ATLAS Collaboration, Eur. Phys. J. C 76 (2016) 642

[5] ATLAS Collaboration, Phys. Lett. B 761 (2016) 136

[6] ATLAS Collaboration, Eur. Phys. J. C 78 (2017) 487
[7] ATLAS Collaboration, Phys. Rev. D 91 (2015) 112013

[8] ATLAS Collaboration, JHEP 11 (2017) 191

[9] ATLAS Collaboration, Phys. Rev. D 98, 012003 (2018)

[10] ATLAS Collaboration, Eur. Phys. J. C 77 (2017) 292

[11] ATLAS Collaboration, arXiv:1802.06572 hep-ex]

[12] ATLAS Collaboration, Eur. Phys. J. C 77 (2017) 220

[13] ATLAS Collaboration, ATLAS-CONF-2018-029 https://cds.cern.ch/record/2628772

[14] ATLAS Collaboration, Eur. Phys. J. C 77 (2017) 531

[15] ATLAS Collaboration, JHEP 01 (2016) 064

[16] ATLAS Collaboration, Phys. Lett. B (2016) 228-246

[17] ATLAS Collaboration, arXiv:1609.03920 hep-ex]

[18] ATLAS Collaboration, JHEP 01 (2018) 63

[19] ATLAS Collaboration, Eur. Phys. J. C 78 (2018) 186

[20] ATLAS Collaboration, arXiv:1806.04667 hep-ex]

[21] ATLAS Collaboration, Phys. Lett. B 780 (2018) 557

[22] ATLAS Collaboration, JHEP 04 (2017) 124

[23] https://twiki.cern.ch/twiki/bin/view/AtlasPublic/TopPublicResults 